\title{
Words of the Dead Chief, The Birth of the Fenian Movement, Parnell to Pearse
}

\section{Pauline Collombier-Lakeman}

\section{(2) OpenEdition \\ 1 Journals}

\section{Édition électronique}

URL : http://journals.openedition.org/etudesirlandaises/1929

DOI : 10.4000/etudesirlandaises.1929

ISSN : 2259-8863

\section{Éditeur}

Presses universitaires de Caen

\section{Édition imprimée}

Date de publication : 30 juin 2010

Pagination : 196-198

ISSN : 0183-973X

\section{Référence électronique}

Pauline Collombier-Lakeman, « Words of the Dead Chief, The Birth of the Fenian Movement, Parnell to Pearse », Études irlandaises [En ligne], 35-1 | 2010, mis en ligne le 30 septembre 2010, consulté le 21 septembre 2020. URL : http://journals.openedition.org/etudesirlandaises/1929; DOI : https://doi.org/ 10.4000/etudesirlandaises. 1929

Ce document a été généré automatiquement le 21 septembre 2020.

\section{cc) (†) (9)}

Études irlandaises est mise à disposition selon les termes de la Licence Creative Commons Attribution

- Pas d'Utilisation Commerciale - Partage dans les Mêmes Conditions 4.0 International. 


\title{
Words of the Dead Chief, The Birth of the Fenian Movement, Parnell to Pearse
}

\author{
Pauline Collombier-Lakeman
}

\section{RÉFÉRENCE}

Donal McCartney, Pauric Travers (eds.), Words of the Dead Chief, Being Extracts from the Public Speeches and Other Pronouncements of Charles Stewart Parnell from the Beginning to the Close of His Memorable Life, [compiled by Jennie Wyse-Power, with an introduction by Anna Parnell], Dublin, University College Dublin Press, 2009 [1892], XXII +179 p., ISBN 978-1-906359-42-3, $20 €$

Marta Ramón (ed.), The Birth of the Fenian Movement, American Diary, Brooklyn 1859, James Stephens, Dublin, University College Dublin Press, 2009, XxIX + 109 p., ISBN

978-1-904558-91-0, $20 €$

John J. Horgan, Parnell to Pearse, Some Recollections and Reflections, Dublin, University College Dublin Press, 2009 [1949], XXXV + 359 p., ISBN 978-1-906359-29-4, $24 €$

1 Destinée à la réédition de textes de références devenus quasi-introuvables (essais, mémoires, journaux intimes ou nouvelles de figures irlandaises connues datant des $\mathrm{XVIII}^{\mathrm{e}}$, $\mathrm{XIX}^{\mathrm{e}}$ et $\mathrm{XX}^{\mathrm{e}}$ siècles), la collection "Classics of Irish History » publiée par les presses universitaires de University College Dublin incluent les trois ouvrages mentionnés cidessus parmi ses plus récentes publications.

Le premier ouvrage, Words of the Dead Chief, publié à l'origine en 1892 par la nationaliste et féministe Jennie Wyse-Power, rassemble des extraits de discours que Charles Stewart Parnell prononça tout au long de sa carrière politique entre 1875 et 1891 . Outil de propagande destiné à célébrer les principes énoncés par Parnell alors que celui-ci venait de décéder après avoir été désavoué par le parti qu'il avait contribué à fonder, cet ouvrage n'en reste pas moins une référence de tout premier plan pour quiconque s'intéresse à Parnell et à l'histoire du nationalisme irlandais. En effet, le leader irlandais a laissé à la postérité bien peu de textes publiés et fait surprenant, il n'existe à ce jour 
aucune édition complète des discours d'un personnage aussi important. L'ouvrage de Jennie Wyse-Power, bien que très lacunaire et partial, est donc encore à ce jour le seul livre offrant un échantillonnage des très nombreuses allocutions que fit Parnell. La réédition entreprise ici par $\mathrm{D}$. McCartney et $\mathrm{P}$. Travers apporte des améliorations nonnégligeables par rapport à la première version de l'ouvrage : les dates des discours sont plus clairement indiquées (l'année figure à chaque fois), les notes sont plus abondantes (41 contre 3) et le texte original est précédé par une introduction critique intéressante pour plusieurs raisons : en effet, elle dresse un portrait de Jennie Wyse-Power et Anna Parnell, deux figures de femmes qui, notamment pour la première, jouèrent un rôle au sein du nationalisme irlandais ; les omissions faites par Wyse-Power dans le choix des discours sont également signalées et commentées et enfin, une analyse des qualités d'orateur de Parnell est proposée.

Le deuxième ouvrage, The Birth of the Fenian Movement, est aussi un document de tout premier plan. Il s'agit d'un journal en deux parties retraçant le séjour aux Etats-Unis que fit entre octobre et février 1859 le fondateur de l'Irish Republican Brotherhood James Stephens. Au cours de son séjour, Stephens tenta de mobiliser les diverses factions du nationalisme irlando-américain et surtout d'obtenir le soutien des exilés Thomas Francis Meagher et John Mitchel afin de récolter des fonds en vue d'une insurrection en Irlande; il fonda également un mouvement américain allié à l'IRB, The Fenian Brotherhood. Le texte dévoile toutes les difficultés d'organisation auxquelles Stephens dut faire face (réunions sans succès, problèmes rencontrés pour rassembler des fonds, etc.). La forme du journal permet également au lecteur de mesurer toute la complexité de la personnalité du leader fenian : tantôt arrogant, cynique voire misanthrope et très critique à l'égard de certaines figures comme Michael Doheny; tantôt enthousiaste et dévoué corps et âme à la cause nationale. À nouveau le travail paracritique effectué par Marton Ramón est à noter: une introduction rappelle les origines du mouvement Fenian suite à l'échec de l'insurrection menée par les partisans de la Jeune Irlande en 1848 et une conclusion revient utilement sur certains aspects du journal: le bilan financier de la mission de Stephens, qui fut finalement plutôt positif ou l'histoire des relations entre Stephens et les nationalistes irlando-américains Michael Doheny et Joh O'Mahony. Le travail de transcription du manuscrit original, les textes introductifs accompagnant chaque partie du journal et les notes abondantes contribuent à rendre le texte plus accessible et les notices biographiques ajoutées en appendice ne manqueront pas d'être des outils précieux pour tout chercheur intéressé par la question du nationalisme irlando-américain.

Le dernier ouvrage, Parnell to Pearse, est légèrement différent des deux précédents dans la mesure où il fut publié postérieurement aux événements qu'il décrit (1949). Si l'ouvrage couvre une période allant des débuts politiques de Charles Stewart Parnell (1875) aux élections législatives de 1918 qui virent la victoire du Sinn Féin et le déclin définitif du parti parlementaire irlandais, la plus grande partie est consacrée à l'après-1900 et notamment aux années qui virent l'introduction du troisième Home Rule Bill (1910-1914) et la radicalisation à la fois du nationalisme irlandais et de l'unionisme. John J. Horgan, avocat et coroner originaire de Cork, propose un récit autobiographique dans lequel il raconte ses engagements en tant que nationaliste dans la vie politique irlandaise, à l'échelle locale et nationale. Ceci lui permet de brosser des portraits de toutes les personnalités qu'il rencontra jusqu'en 1918 (entre autres, D. P. Moran, Edward Martyn, John Redmond, T. P. O'Connor, Thomas Kettle, William Redmond, Patrick Pearse et Eamon de Valera). On trouve aussi des passages 
intéressants sur la vie politique locale à Cork, notamment ceux concernant les campagnes électorales qui opposèrent les partisans du parti parlementaire irlandais de ceux de William O'Brien, en rupture définitive avec le parti à partir de mars 1909. Farouche partisan de John Redmond et de son esprit de conciliation, Horgan proposa des solutions à la question d'Irlande qui ne furent pas retenues mais qui témoignent de l'esprit de compromis qui caractérisait la tradition du nationalisme constitutionnel irlandais : un droit de veto absolu accordé à la province d'Ulster, représentée au sein du Parlement et du gouvernement irlandais (1916), un statut politique pour l'Irlande qui lui permette de rester dans l'Empire britannique tout en jouissant d'une autonomie législative sur le modèle de l'Afrique du Sud («Dominion Home Rule »,1917) ou encore une alliance avec les éléments modérés du Sinn Féin afin de faire valoir l'autonomie de l'Irlande à la Conférence de Paix prévue suite à la fin de la Première Guerre mondiale (1918). 